\title{
Automating Hostel Telephone Systems
}

Rohan Prabhu Murje, Bhaskar Rishab, Krishna Gopalrao Jorapur, MuccatiraThimmaiah Karumbaiah, Muddenahalli Nagendrappa Thippeswamy*

Department of Computer Science and Engineering, Nitte Meenakshi Institute of Technology, Yelahanka, Bengaluru, India-560064

\begin{tabular}{l} 
A R T I C L E I N F O \\
\hline Article history: \\
Received: 22 April, 2018 \\
Accepted: 01 June, 2018 \\
Online: 18 June, 2018
\end{tabular}

Keywords:

Communication

Digital lines

EPABX systems

PRI cards

\section{Introduction}

The business phone systems are more affordable due to the rise of cloud technology. This has transferred the telephone industry standards [1]. Automated phone systems can manage a high volume of callers and provide a method for filtering calls in a timely manner for small business [2]. The automation can save a substantial amount of time and money [2]. The number of parents opting for a residential school for their kids is on the rise, parents expect the right atmosphere for the students to develop. The schools do their best to provide the best facilities but often fail to monitor and control the usage of these facilities. One of the most common problems faced by the institutions is manually monitoring telephone facilities provided to the students. Parents often complain about students not contacting them on a regular basis. There are many other problems, for example, not everyone gets a chance to call; unlimited talk time calls are made to unauthorized people, and unauthorized access to the facility [3]. In order to counter all these problems, an automation solution which revolves around the automation of controlling and monitoring the calls that are made is proposed. It has pre-set numbers, pre-set call time, automated duration control and many other features which help in

\footnotetext{
*Thippeswamy M.N., Department of Computer Science and Engineering, NitteMeenakshi Institute of Technology, Bengaluru, India-560064, 00919686329815, Email: thippeswamy.mn@nmit.ac.in
}

reducing the amount of human intervention required. This is done by setting up a telephony network comprising of PRI lines, an interface card, an EPABX system and analogue phones. The backend software controls the communication flow allowing us to manage and monitor every aspect, with minimal human intervention.

\section{Related Work}

The automatic voice responding system that uses the computer stored data is proposed in [4]. When a parent dialed the hostel phone number, they will get the answer in automatic stored voice form in this work. In [5], when a parent dialed the specified mobile number, they will get the student percentage attendance in voice form. Thus, parents can get their ward performance report from anywhere at any time.

As the rate at which residential institutions are growing, it is found that there is a greater need to automate the telephone calling system. It is necessary for a student to be in constant touch with his/her parents to remain motivated. The ability to quickly and efficiently manage hundreds of simultaneous calls is necessary in order to effectively manage the process [6]. 


\section{M.N. Thippeswamyet al. / Advances in Science, Technology and Engineering Systems Journal Vol. 3, No. 3, $147-151$ (2018)}

In this paper, different methods are explored that have been used over the years to provide this facility. In order to facilitate the calling process, one approach is to use coin phones. Here, each student can call their respective parents by inserting coins into the phone and making calls. This isn't feasible as there can be no control and monitoring of the system as the number of students increase. The other method that has been implemented is to allow only incoming calls using an analogue line connected to an analogue phone, the incoming call can be received by the student and the facility can be used.

The provision to block the call during the closed hours is provided, avoiding unauthorized access to the facilities. Though this system can address a lot of problems it cannot address some major issues like monitoring incoming calls, controlling the access and since analogue lines are used, only one call can be made/received at a time; hence, giving rise to a new problem of investment on analogue lines and cabling. Similar telephony systems are used for intra-communications within organizations, they use similar methods to allow intercom.

An EPABX system [7] with advance feature allows staff members to monitor the telephone usage and manage calls over a large organization. In this paper, analogue line is used instead of PRI lines [8]. Such systems are also used in a Call center where an Omni channel solution is required.

Compared to the existing system of telephone management, aproposed system uses PRI lines and a PRI interface card which is connected to the system, where the server controls the needs of the institution whereas generally there is no involvement of a local server providing this functionality. The systems are only used for intercom and other such facilities hence functions like preassigning call timings are not present.

\section{Novel Contribution}

This solution allows monitoring and control of a student's telephone activities in the following ways:

i. Pre-assigned call timings, the lines only open during this time, hence avoiding unauthorized access.

ii. Pre-assigned Phone numbers, Speed dial is set to these telephone numbers, hence avoiding students calling other unknown numbers.

iii. Pre-assigned authorisation code, each student has an authorization code, which activates their account.

iv. Pre-assigned call duration, flexible call duration, admin can set call duration.

v. Automatic report generation, reports are sent every day to the administrator's mail ID.

vi. Reports based on status of the call, reports based on call status, i.e. if a call is connected or dropped, can be generated.

vii. History of call log, complete call history of the student can be checked with few clicks. viii. Flexible call charges, the management has the privileges to charge for the facility provided.

\section{System Design}

The system consists of different telephony hardware, this is put-together with backend software coded in asterisks and Hypertext Pre-processor (PHP). Also the system consists of database tables created in My Structured Query Language (MySQL). The main components of the system are PRI lines, PRI card, EPABX system and a local server. The telephone systems architecture consists of various hardware devices.

This software is built on controlling the PRI lines which can allow up to 30 simultaneous calls; this digital line is connected to the local server which consists of a PRI card. This card allows the PRI lines to be connected to the server (refer to Figure 7). In this paper, for the outgoing lines, an EPABX is used, which is a branch exchange device that helps to channel the outgoing calls. PRI line is also known as a T1 line, it is a multi-channel line. The feature of a call transferring and forwarding is another area enabling mobility of the users. Auto conferencing and automatic redialling of numbers found engaged on the first trial are some of the other advancements in the features of the EPBAX. An EPABX also checks the traffic and is also called as a switching system.

\subsection{A brief functionality of each component:}

- PRI Lines: PRI lines or primary interface lines are digital lines, which are a telecommunication standard that enables traditional phone lines to carry voice, data and video traffic, among others. A PRI line physically has only one line terminating on the interface but can allow us to send or receive 30 calls simultaneously. A PRI circuit consists of two pairs of copper lines terminating on a modem from a service provider premises to the customer premises. It uses multiplexing/de-multiplexing techniques to carry more than one channel in a single circuit. There are two common types of PRI lines used namely E1 and T1. The E1 type consists of 30 channels used mostly in India and Europe. Type T1 consists of 23/24 channels. T1 type is mostly used in the US. In this paper, the PRI E1 type is used.

- PRI interface cards: A PRI Card is used to connect PRI lines to IP PBX/ IP Telephony Server so that the entire Analog phones (extensions) can make outgoing calls or receive incoming calls using it. This is inserted in a local server in the Peripheral Component Interconnect (PCI) 3.3V/ PCI 5V/ PCI Express slots. These cards can have one slot, two slots or four slots.

- EPABX System:An EPABX system which is a branch exchange device that helps channeling the outgoing calls. It also controls the traffic and is also called a switching system.

- Analog Phones:The analogue phones allow speed dialing, hence it can serve the purpose of not allowing dialing of numbers and pre-assigning telephone numbers.

- Local Server:The local server consists of the software which controls communication. This software has the following modules. 
- Student details:This module contains all the data of the students, it contains a database which stores the name, id, auth code and three pre-assigned telephone numbers.

-Verification module: This module contains the logic which verifies the authentication of the student. The number entered on the analog phone is matched with the authentication code present in the database; the student details present under the entered auth code is loaded onto the speed dial of the telephone.

- Balance account: Here all the students account balance is checked to see if an outgoing call can be made, if not then a voice over message is played alerting the user to recharge their account. After the call terminates the latest balance is updated to the database.

- Timings module: This module contains the assigned calling time. When the phone is accessed the timing, module is accessed and the time allotted is matched with the current time, if the there is a match between current time and opening time the user is allowed to use the facility else a voice over message alerts the user about the line being closed.

- System module:The system architecture consists of the above mentioned hardware. First, the PRI line is terminated at a Local Area Network (LAN) switch and a LAN cable is drawn to the local server which has a PRI card. In the local servers various modules are present. From the local server a branch exchange is used and then physical wiring is done to each room of the hostel which has a telephone.

\section{Implementation and Results}

The solution has been implemented using PHP as a front-end for the user interface and asterisks coding is used as a back-end to control the communication process. The implementation is performed by setting up telephony hardware as follows: the PRI line is terminated at the PRI interface card, which is present in our local server. In the local server the software to control and monitor the system is locally hosted. There are LAN connections between the local server and the EPABX system, from the EPABX system there are physical wiring to the hostel room where the phones are present.

This section shows the results that have been obtained from the implementation of this solution. The Figure (s) $1-6$ are the results obtained for the proposed system.

The User Interface (UI) of the webpage is shown in Figure 1, which prompts the admin to login to the system. Upon successful login the admin can access different features present like the student details page depicted in Figure 2, here an authorization code is given to each student and their outgoing telephone numbers are assigned.

The next module is the timings module which is shown in Figure 3, here the admin can allot timings for outgoing and incoming calls for everyday of the week.

\section{LOGIN}

Welcome! Please enter your login details.

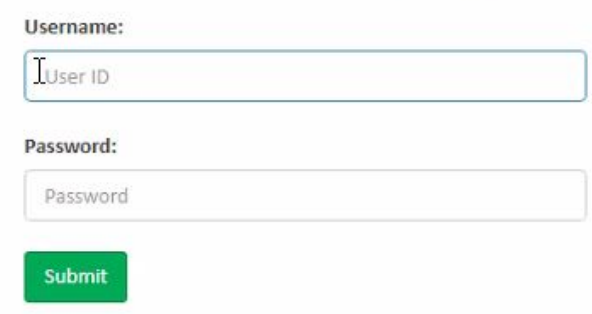

Figure 1: Shows the login page.

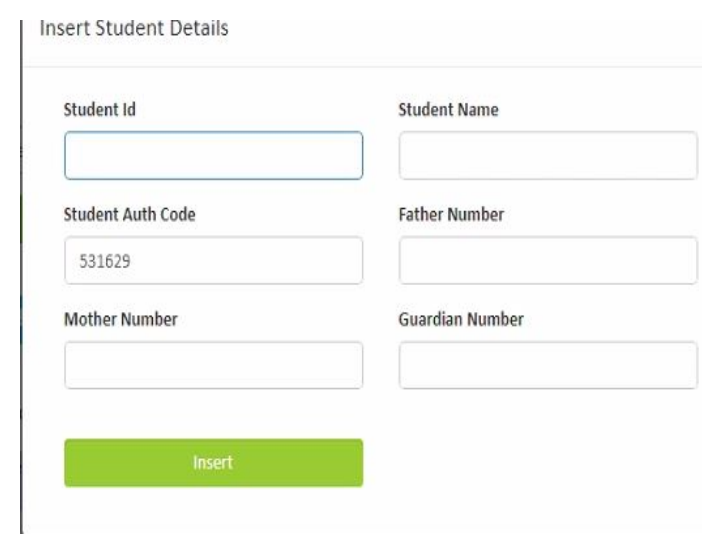

Figure 2: Represents the screen for entering student details.

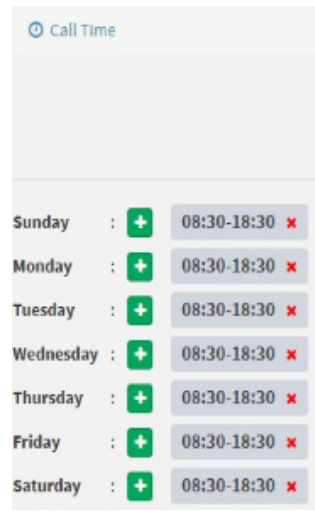

Figure 3: Shows the call timing module.

Enter The Recharge Amount

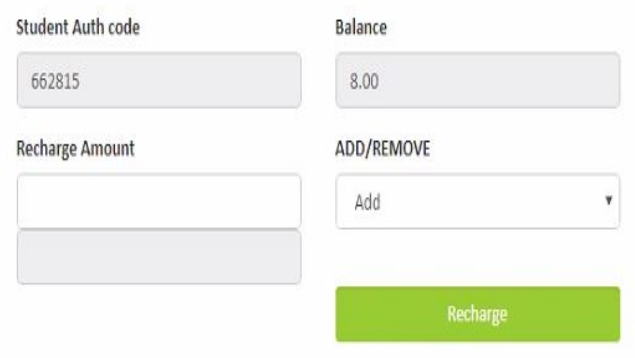

Figure 4: Shows the recharge module of the software 
To make an outgoing call the admin has to recharge the students account, this is depicted in Figure 4. Once an outgoing call is made and is disconnected the database is updated and reports are generated as seen in Figure 5 (a) and (b). These bills are generated based on the flexible call charges module, which allows the management to charge over the existing price, this module is depicted in Figure 6.

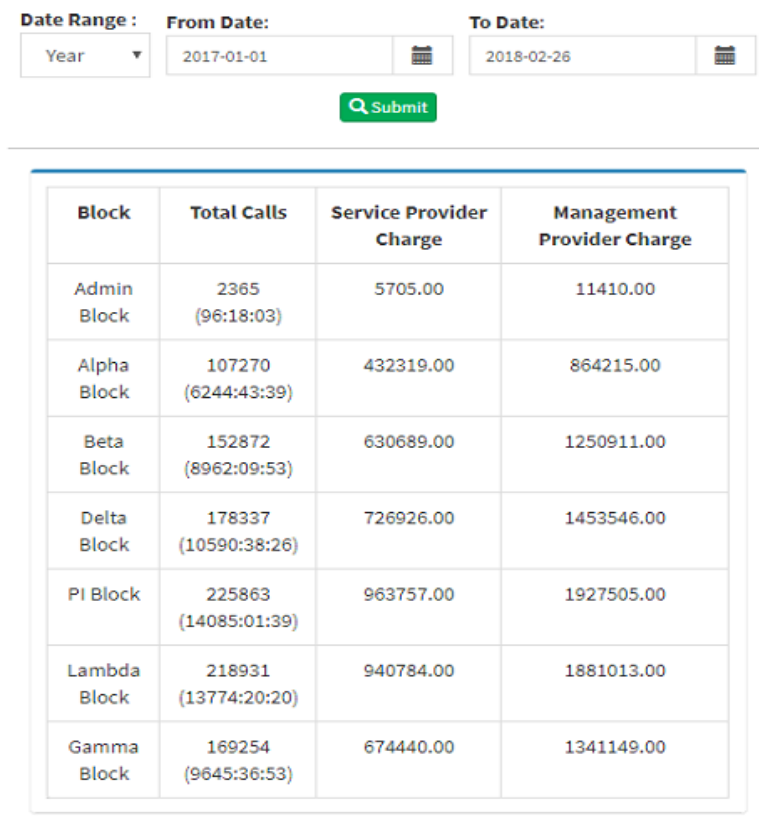

(a)

\begin{tabular}{|l|l|}
\hline Total Calls - (1054892) & Total Duration - (63398:48:53) \\
\hline Service Provider Charges & 4374620 \\
\hline Our Charges & 8729749 \\
\hline Our Profits & 4355129 \\
\hline
\end{tabular}

(b)

Figure 5: Shows the billing reports over a period of time.

\section{Conclusion}

This paper explains the working of a telephone system, which can help an institution tremendously by automating the control and monitoring of the process. This reduces the human effort that is required to monitor each and every call made by hundreds of students on a daily basis. This solution saves a lot of time and the effort required to monitor each and every call of hundreds of students. Reports are generated every day based on the status of the call, last call charge, total profits giving the management a bird's eye view of the process. Adding to the ease of control and monitoring it also gives the institution flexibility to charge per unit call for example, if the call provider charges Indian Rupees (INR) 1.0 per minute, the institution has options to charge INR 1.5 per minute, or INR 2.0 per minute or any amount they would want to. This solution would be beneficial for institutions with greater than hundred students who have taken residential facility from the school.

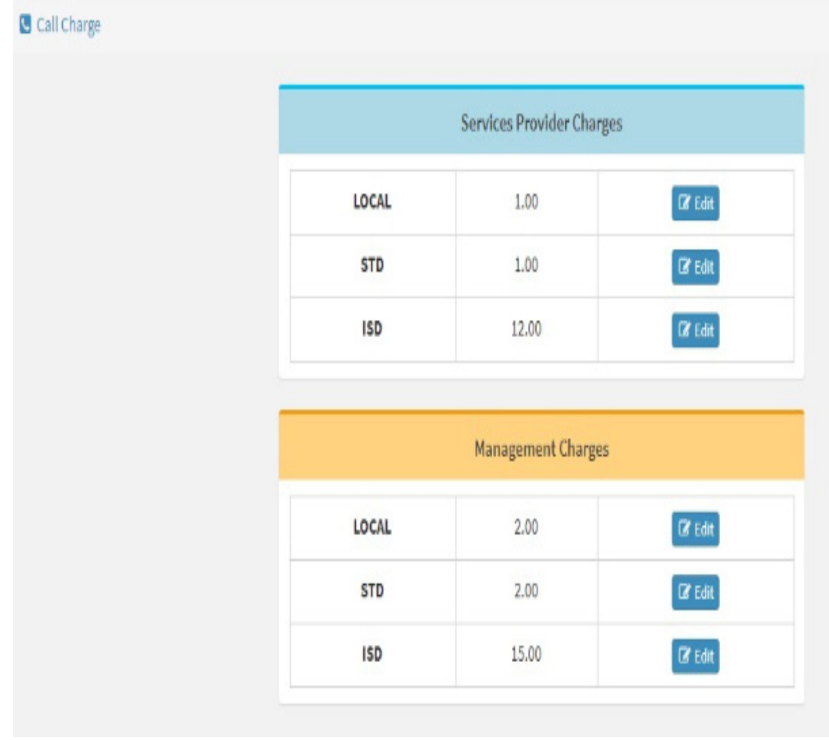

Figure 6: Shows the call charges module.

\section{Conflict of Interest}

The authors declare no conflict of interest.

\section{References}

[1]. BT Prepare to Switch to an All IP Network by 2025, [online] Available: http://www.sa1 solutions.com/blog-bt-moving-ip-network.php.

[2]. A. Smith, Automated Phone Systems: Identifying the Problem with yours and how to turn the ship around, [Online] Available: https://www.theivrvoice.com/automated-phone-systems/

[3]. D. Kirk, How much is intelligent automation saving you, [online] Available:https://www.forbes.com.

[4]. I.J.Vinila, "Voice Response System for Parents of Hostel Students", International Journal of Innovative Research in Computer and Communication Engineering, Vol. 4, Issue 5, May 2016.DOI: 10.15680/IJIRCCE.2016. 0405022

[5]. J. Patil and J. Barole, "Automatic Voice Responding System for Parents of Students", International Journal of Innovative Research in Science, Engineering and Technology,Vol. 4, Issue 6, June 2015.

[6]. Hostel Telephone [online] Available:http://mipsoft.com/telephone.html

[7]. U. Nadeem. "The Functions of a PBX", [Online] Available: https://www.lifewire.com/functions-of-a-pbx-3426325

[8]. K. Rajesh, Telecom Trunk Lines: Difference Between Analog Line PRI/E1/T1 Digital-Line-GSM-Gateway, [online] Available: http://www.excitingip.com/2111/telecom-trunk-lines-difference-betweenanalog-line-prie1t1-digital-line-gsm-gateway/ 


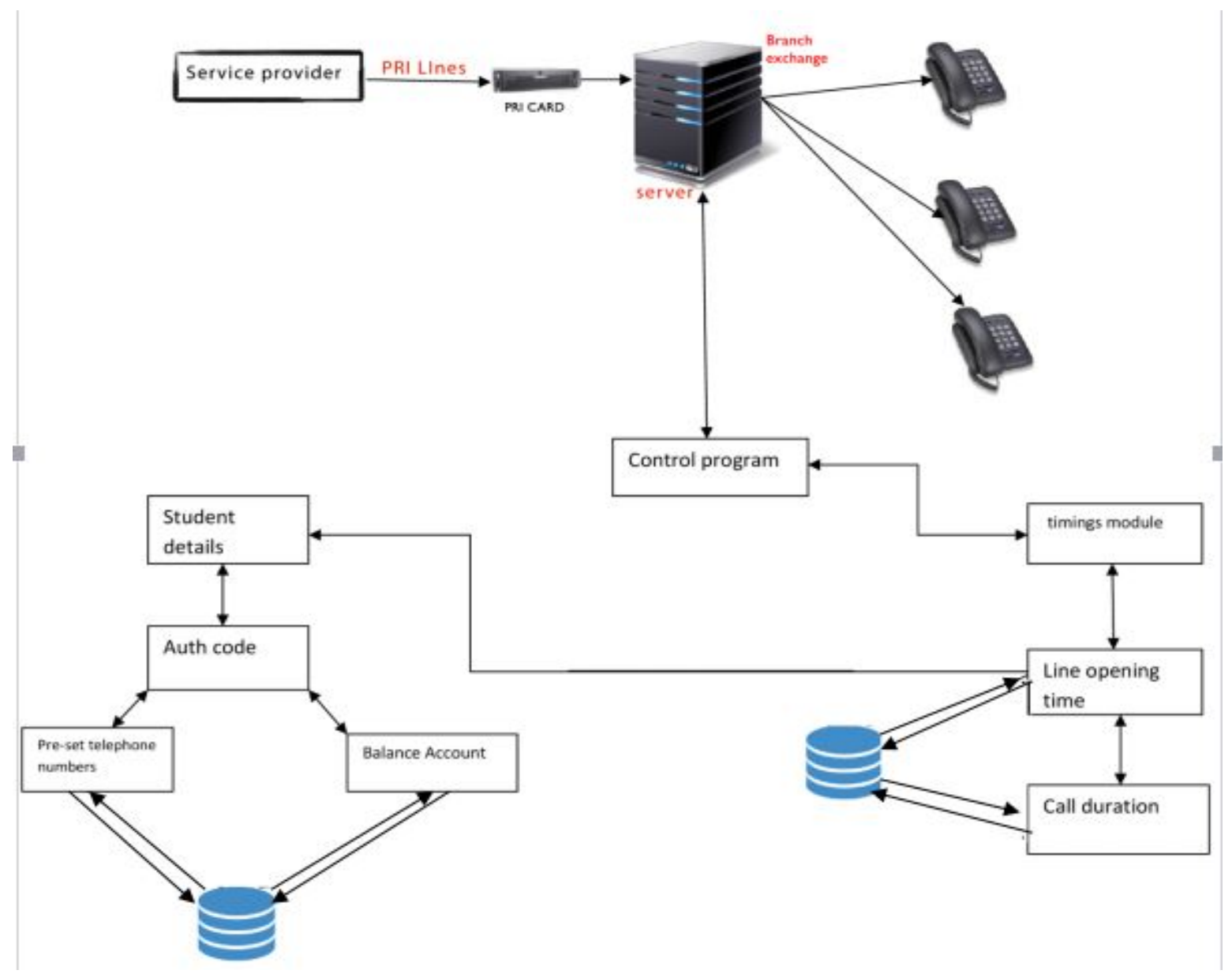

